\title{
COMPARATIVE STUDY AND ANALYSIS OF BER OF CDMA FOR DIFFERENT MODULATION SCHEME
}

\author{
Arun Kumar, Dr. Manisha Gupta \\ Research Scholar: Dept of ECE, JECRC UNIVERSITY, Jaipur \\ Arun.kumar1986@live.com \\ Associate Professor, Dept of Physics, JECRC UNIVERSITY, Jaipur \\ Manisha.gupta@jecrcu.edu.in
}

\begin{abstract}
:
With the increasing demand in wireless communication, it has become necessary to give better and efficient service to users by using better technique. This paper propose and analyse the bit error rate of CDMA, for different modulation schemes such as BPSK, QPSK and QAM. By Choosing a reliable modulation scheme and better filtration Technique the enhancement of the performance can be obtained in transmitter and receiver of CDMA system. Simulated result is shown to analyse and compare the performance of these systems by using additive white Gaussian noise channel (AWGN).Finally the different modulation schemes is compared on the basis of BER and best modulation scheme is determined. From analysis of three modulation techniques, the system could use more appropriate modulation technique to suit the channel quality, thus we can deliver the optimum and efficient data rate to mobile terminal.
\end{abstract}

Keywords: CDMA (Code Division Multiple Access), QPSK (Quadrature Phase Shift Keying), BPSK (Binary Phase shift Keying), BER (Bit Error Rate)

\section{Council for Innovative Research}

Peer Review Research Publishing System

Journal: INTERNATIONAL JOURNAL OF COMPUTERS \& TECHNOLOGY

Vol 12, No. 8

editor@cirworld.com

www.cirworld.com, www.ijctonline.com 


\section{INTRODUCTION}

Code Division Multiple Access (CDMA) is well known air interface technologies in modern mobile communication. This work proposes and analyzes the bit error rate of CDMA, for different modulation schemes such as Binary Phase shift Keying (BPSK), Quadrature Phase Shift Keying (QPSK) and Quadrature Amplitude Modulation (QAM). By Choosing a reliable modulation scheme and better filtration Technique the enhancement of the performance can be obtained in transmitter and receiver of CDMA, system [1]. To analyze and compare the performance of these systems simulation may be used by using additive white Gaussian noise channel (AWGN) [2]. The technologies like CDMA has increased the standards for wireless communication system in terms of high data speed, mobility both for short range and long range communication. With the increasing uptake of so-called smart phones in recent times, it has been repeatedly identified that state-of-the-art cellular networks are fast being rendered incapable of consistently delivering the excessively high throughputs demanded by the Internet-savvy users of such devices [3]. If we're all to use our mobile devices to work and play anywhere, we want to access to streaming services and all our own "stuff", instantly, on devices as small as a Smartphone or as large as the screen in an auditorium - properly formatted for the size of the screen, of course. We're already socially networked, 24 hours per day, 7 days a week [4].Till today modulation is only defined as: "process of sending the message with the help of carrier" but now the efficiency, data-rate of communication depend upon on the choice of modulation scheme. So it is essential that we should know the advantage and disadvantage of different modulation scheme in wireless communication system so that one can adopt the efficient one to improve the communication system. Although much of the work is not done focusing on the modulation scheme so here we proposed a work that will analyse the Transmitter and Receiver of CDMA by using different modulation techniques by defining BER, Scatter Plot of Transmit Signals and Received Signal.

\section{Review}

CDMA technique is based on the spread spectrum communication. The CDMA with existing modulation Technique have disadvantage that the presence of a strong interferer can raise the noise significantly for other channels which can cause communication to be halt under serious condition and also it need huge amount of power which result in implementation of extra hardware to adjust the power requirement. For a spread spectrum signal the transmission bandwidth is much wider than the bandwidth of the original signal [5]. A work done by Ghanim et.al shows that QAM gives less bit error rate that makes CDMA more flexible and suitable for mobile communication next generation technology [6]. Recently simulation performed by Arun et.al shows that in a CDMA transmitter, the information is modulated by a spreading code, and in the receiver it is correlated with a replica of the same code. Thus, low cross-correlation between the desired and interfering users is important to suppress the multiple access interference. Good auto-correlation properties are required for reliable synchronization and reliable separation of the multipath components. Having good auto-correlation properties is also an indication of good randomness of a sequence, which allows us to connect other important sequences properly and QPSK is better modulation scheme for CDMA [7]. CDMA Access is a new technology used in wireless communication devices. CDMA gives us secure and reliable based calls [8]. A review done by Bhardwaj et.al[9] Shows over the years researchers have sought ways to extend the user capacity of CDMA systems either by employing optimum [maximum likelihood (ML)] detection, interference cancellation (IC) methods, or other methods such as the de-correlating receiver. CDMA is analogous to the last example where people speaking the same language can understand each other, but not other people. Similarly, in radio CDMA, each group of users is given a shared code. Many codes occupy the same channel, but only users associated with a particular code can communicate [10]. CDMA is the platform on which $2 \mathrm{G}$ and $3 G$ advanced services are built. It is the fastest growing wireless technology and it will continue to grow at a faster pace than any other technology [11]. CDMA can effectively reject narrow band interference. Since narrow band interference affects only a small portion of the spread spectrum signal, it can easily be removed through notch filtering without much loss of information. CDMA devices use a rake receiver, which exploits multipath delay components to improve the performance of the system. In a CDMA system; the same frequency can be used in every cell, because channelization is done using the pseudo-random codes. Reusing the same frequency in every cell eliminates the need for frequency planning in a CDMA system; CDMA systems use the soft hand off, which is undetectable and provides a more reliable and higher quality signal [12]

\section{PROPOSED METHODOLOGY:}

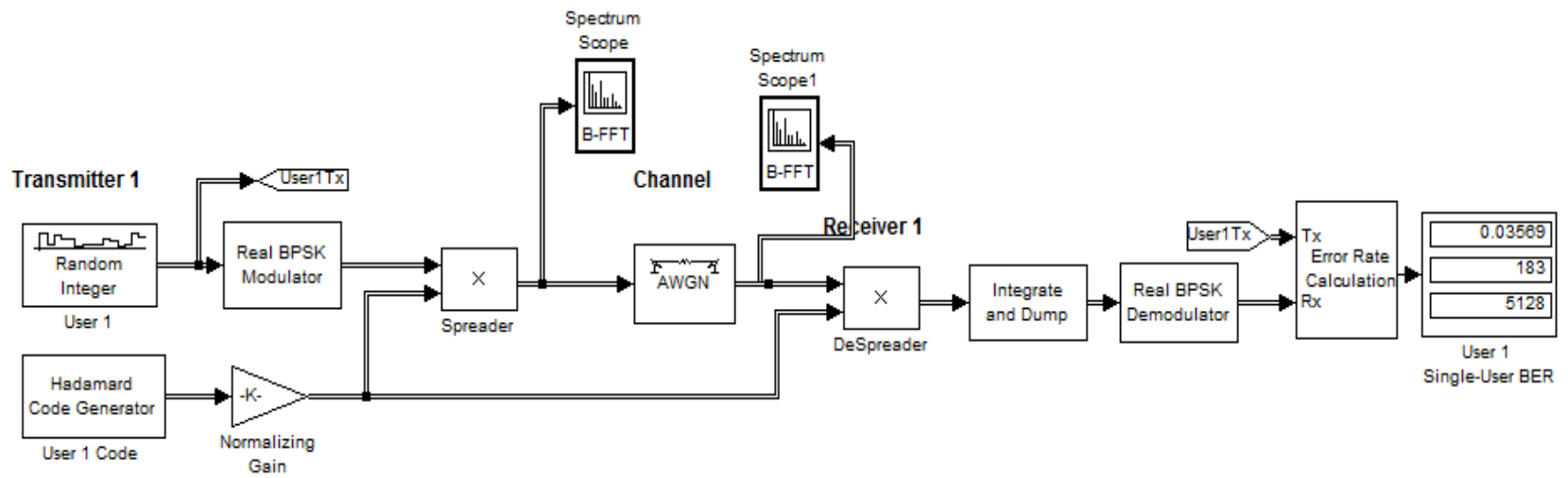

Fig 1. Methodology for CDMA Using BPSK Modulation Technique: 


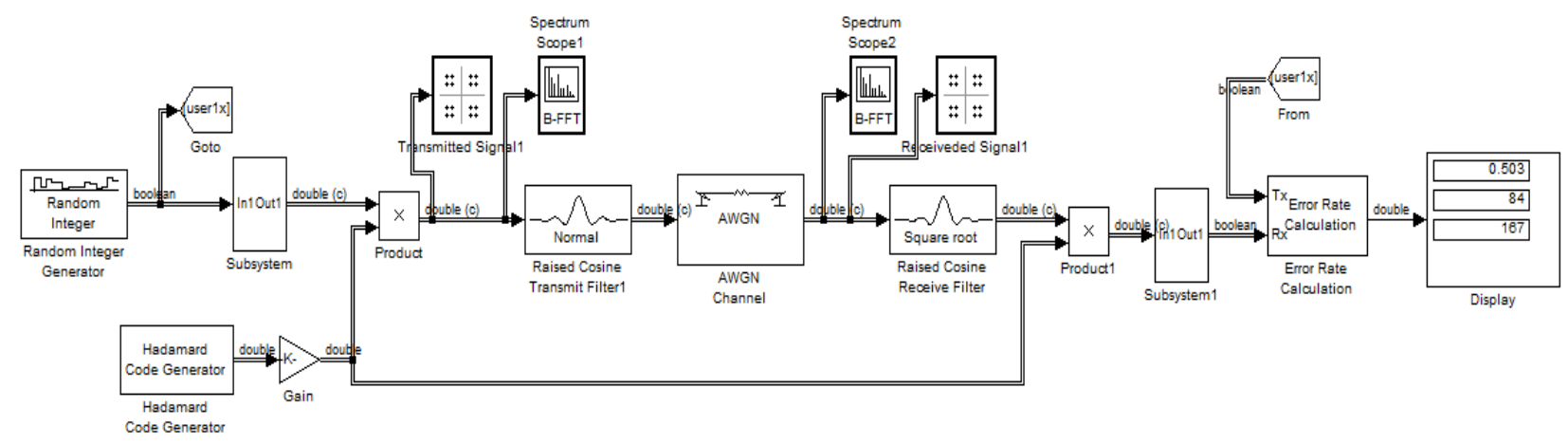

2. Methodology for CDMA Using QPSK Modulation Technique.

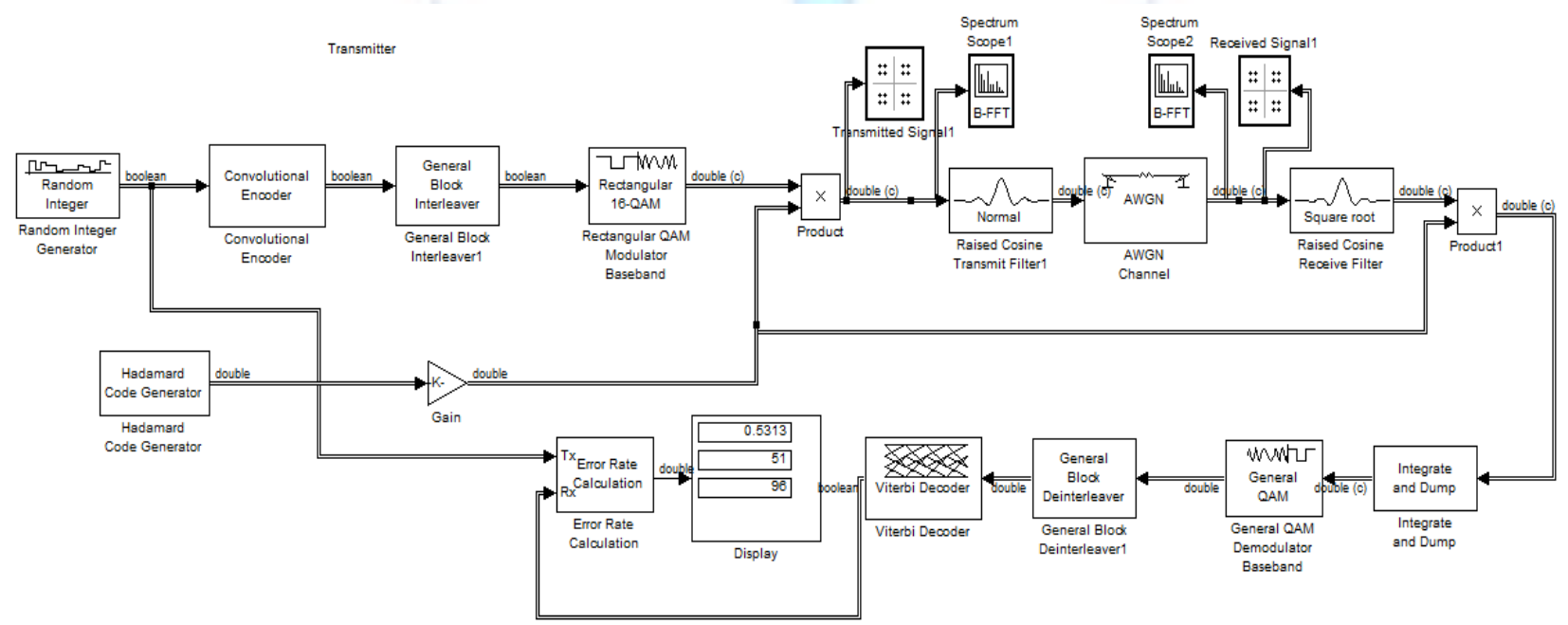

Fig 3. Methodology for CDMA Using QAM Modulation Technique

\section{RESULTS:}

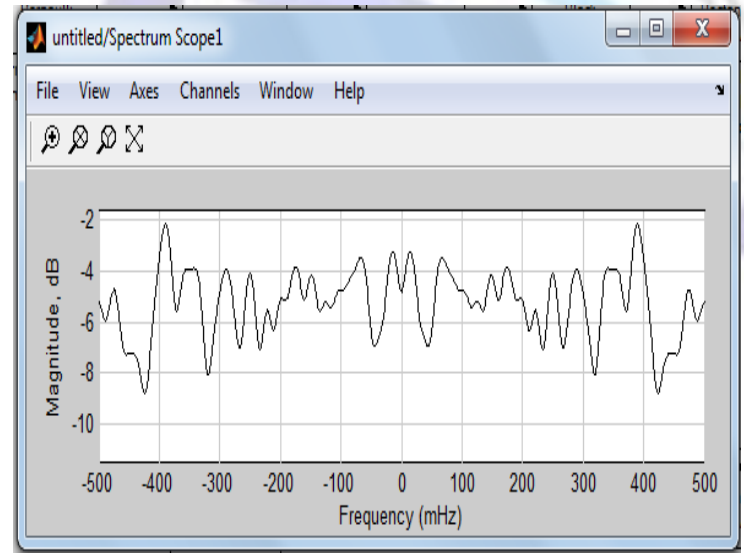

Fig 4. Transmitted Signal BPSK in CDMA

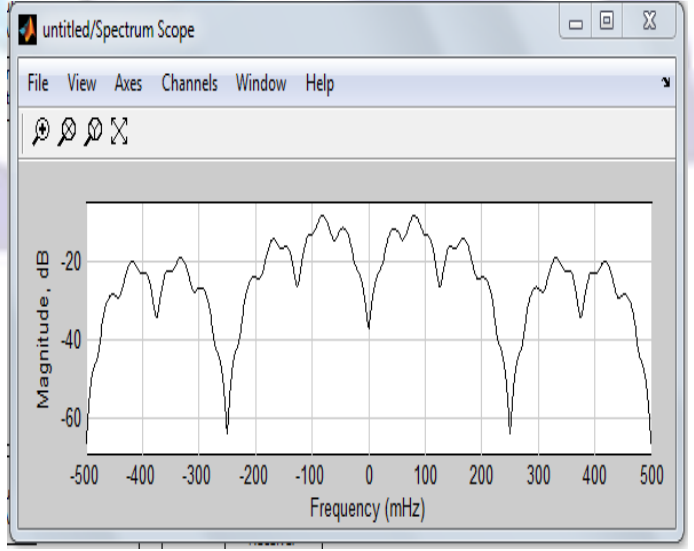

Fig5. Received Signal for BPSK in CDMA 

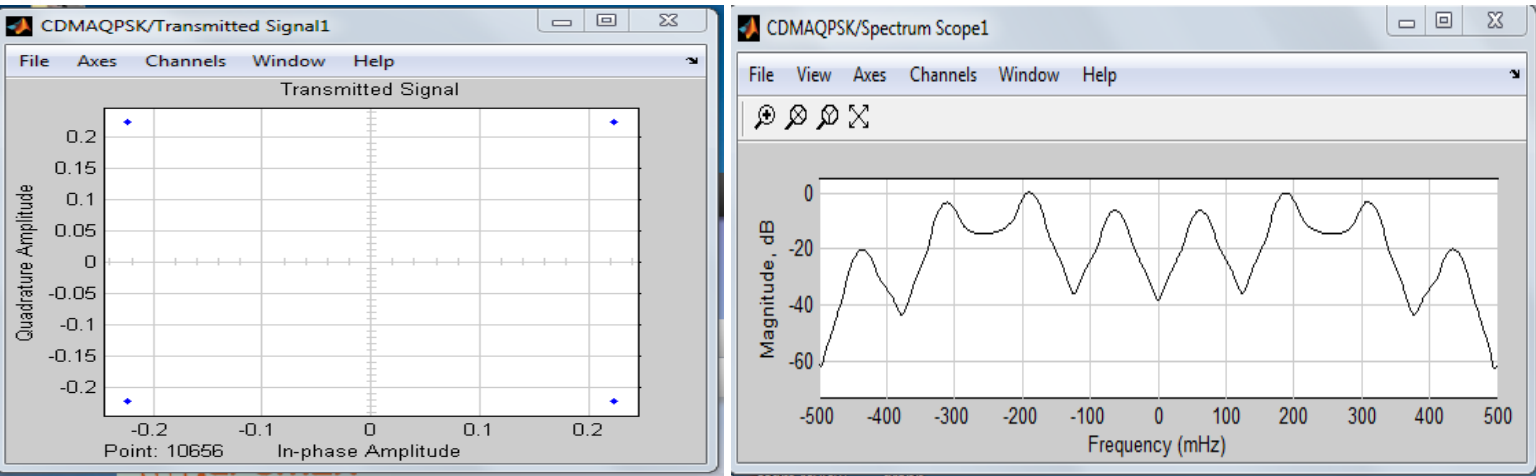

Fig 6. Transmitted Signal \& Scatter plot of Transmitted signal For QPSK in CDMA.
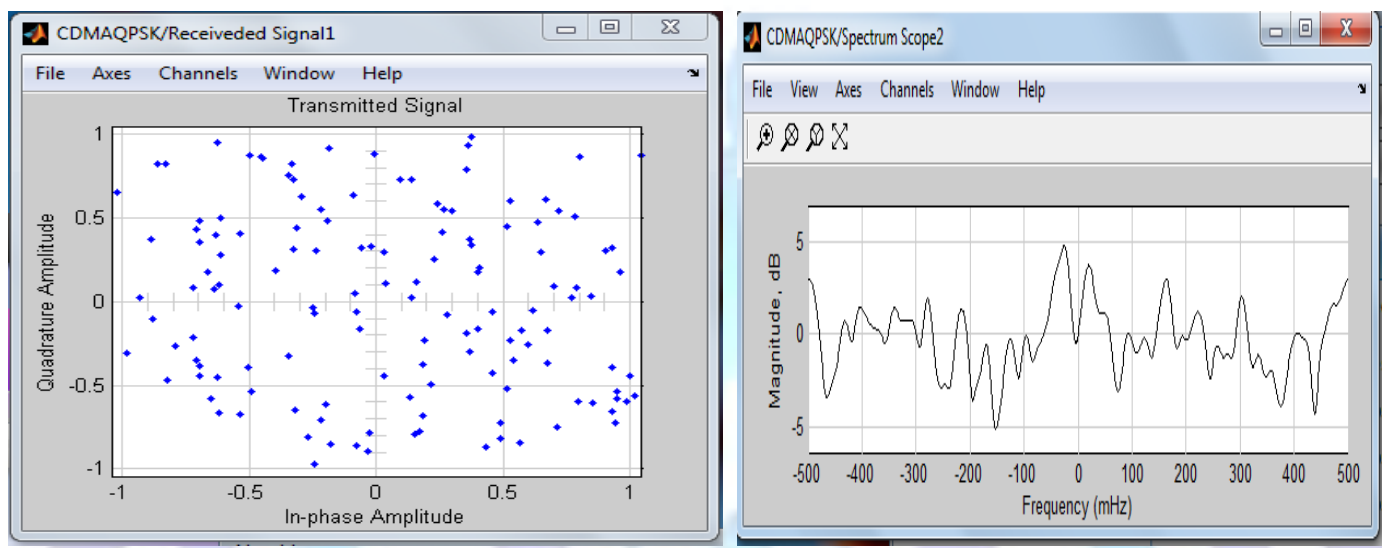

Fig 7. Received Signal \& Scatter plot of Received Signal for QPSK in CDMA
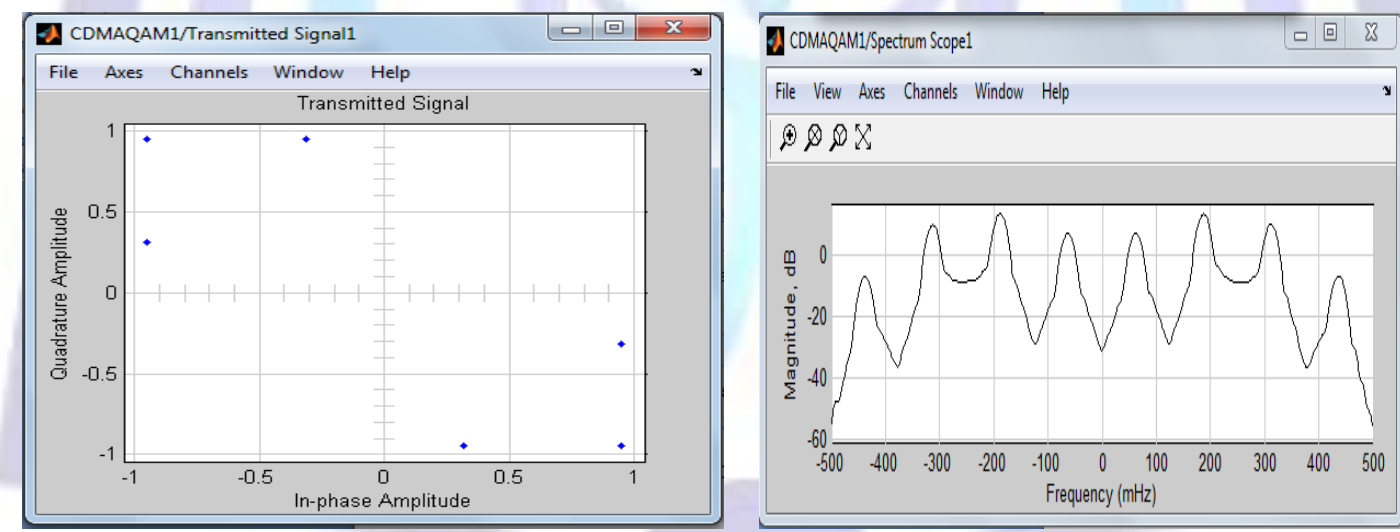

Fig 8. Transmitted Signal and Scatter plot of Transmitted signal for QAM in CDMA.
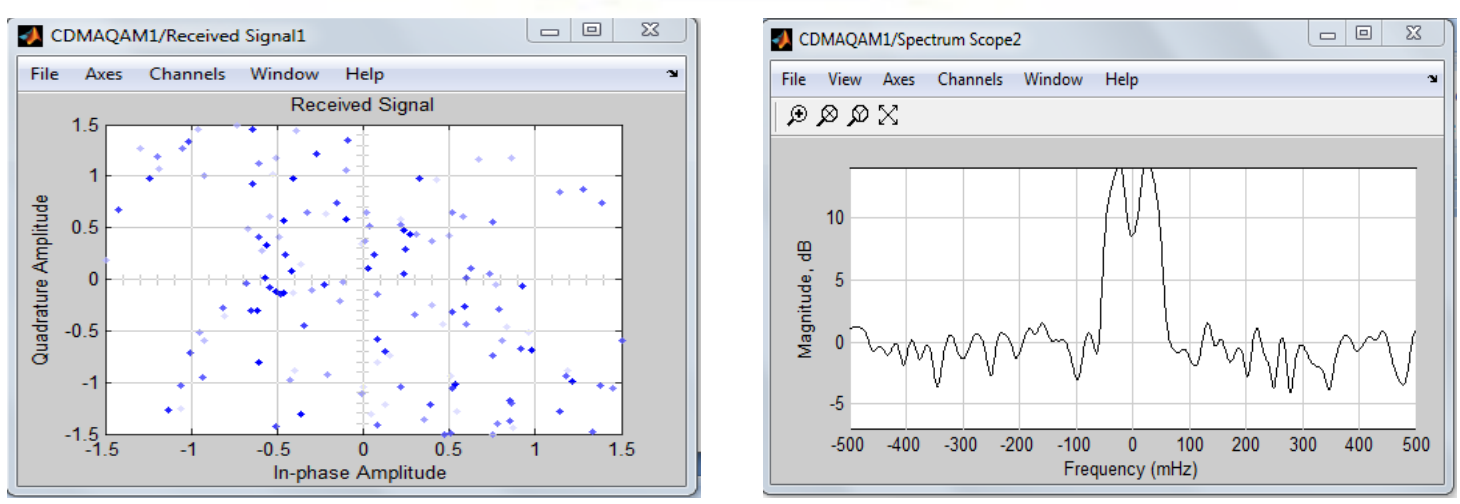

Fig.9. Received Signal \& Scatter plot of Received signal for QAM in CDMA 


\section{CONCLUSION:}

The simulation of different modulation Technique has been generated and compared. From the comparison we conclude that BPSK modulation is best modulation scheme for CDMA system with less BER as compared to QAM and QPSK.

\section{REFERENCES:}

1. Singh M., Schdeva S., Arora A., Singh U., "Analysis of CDMA by Using Simulink", International Journal of Electronics and Computer Science Engineering ,1(3), 384-392, (2011), ISSN: 2277-1956.

2. Yun M., Rong Y., Zhou Y., Choi H., Kim J., Sohn J., Coi J., "Analysis of Uplink Traffic Characteristics and Impact on Performance in Mobile Data Networks", IEEE International Conference on Communications (ICC), Beijing , 3-13, (2008).

3. Measuring the Information Society: The ICT Development Index. InternationalTelecommunicationUnion,UK, www.itu.int/ITUD/ict/publications/idi/material/2009/MIS2009_w5. pd.

4. Patil S., Patil V., Bha P., "A Review on 5G Technology", International Journal of Engineering and Innovative Technology, 1(1), 26-30. ISSN: 2277-3754

5. Ghanim M., Abdullah M., "Analysis of MC-CDMA System in Mobile Communications", I.J. Information Technology and Computer Science, 12(2), 87-94, (2012).

6. Kumar A., Naznin R., "Simulation of Different Modulation Technique in CDMA System using AWGN channel", International Journal Of Computer \& Technology, 1(3), 2346-2354, (2013), ISSN: 2277-3061.

7. Verdu S., "Minimum Probability of Error for Asynchronous Gaussian Multiple-access channels", IEEE Trans. Inform. Theory, (32), 85-96, (1986).

8. Bhardwaj M., "International Organization of Scientific Research Journal “, 1(1), 07-11, 2278-1684, (2012).

9. Awasthi A., Singh D., Srivastava V., "Simulation of QPSK Modulation Technique in CDMA System Using Rician Channel", Conference on Advances in Communication and Control Systems, Atlantic Press, 459, (2013)

10. Kumar A., suman, Renu, "Comparision of $3 G$ Wireless Networks and 4GWireless Networks", International Journal of Electronics and Communication Engineering, 6(1), 1-8, (2013). ISSN 0974-2166.

11. Google, "www. wirelessblog spot. in. /HTML". 